\title{
Du nouveau dans le traitement de la sclérose latérale amyotrophique
}

La sclérose latérale amyotrophique (SLA, maladie de Charcot ou de Lou Gehrig suivant la rive de l'Atlantique sur laquelle on se trouve) est une atteinte dégénérative des motoneurones qui touche les adultes. En dehors de quelques formes familiales pour lesquelles on a trouvé une mutation de la superoxyde-dismutase $\mathrm{Cu} / \mathrm{Zn}(\mathrm{m} / \mathrm{s}$ $n^{\circ} 4$, vol. 9, p. 469), et de la maladie de Guam dans laquelle l'action d'un toxique a été identifiée $\left(m / s n^{\circ} 8\right.$, vol. 3, p. 496), on n'en connaît pas l'étiologie. Les hypothèses sont nombreuses et assez variées mais aucune ne prévaut réellement aujourd'hui. Les travaux de ces dernières années sur la toxicité du glutamate, "l'excitotoxicité " dont médecine/sciences a régulièrement l'occasion d'entretenir ses lecteurs, ont toutefois suggéré que le glutamate et son récepteur-NMDA pourraient être impliqués dans des mécanismes, sinon ubiquitaires, du moins largement répandus, conduisant à la mort neuronale dans diverses maladies neurodégénératives. Voie finale commune potentielle de différents processus provoquant la neurodégénérescence, l'excitotoxicité liée au glutamate a évidemment intéressé au plus haut point les pharmacologues. Les premières molécules antiglutamatergiques produites, capables notamment de bloquer le récepteurNMDA, ont été des outils pharmacologiques très importants, mais elles se sont révélées trop toxiques pour être d'usage thérapeutique. Le riluzole (2-amino-6-(trifluorométhoxy) benzothiazole), mis au point par les laboratoires Pharmuka (aujourd'hui Rhône-Poulenc Rorer), pourrait être l'instrument du passage de molécules antiglutamates à la thérapeutique car il ne présente pas les mêmes effets secondaires et semble, d'après les résultats récemment publiés par Bensimon et al. [1], capable d'interférer avec les mécanismes de la mort neuronale dans la SLA.

Le riluzole a été tout d'abord testé au début des années 80 comme antiépileptique. Il s'était en effet révélé capable de prévenir les convulsions induites chez le rongeur par des électrochocs, les inhibiteurs de synthèse du GABA ou l'ouabaïne, les crises audiogéniques déclenchées par le bruit chez certaines souris et celles liées à la stimulation lumineuse intermittente chez le babouin papio-papio[2]. L'origine de cette action anticonvulsivante ne résidait pas, cependant, dans un effet sur des antagonistes du GABA, comme pour d'autres traitements, mais dans une action antagoniste vis-à-vis de la neurotransmission par certains acides aminés excitateurs [3]. Il était évident que cette particularité devait attirer à nouveau l'attention lorsque l'excitotoxicité du glutamate devint l'ennemi public $n^{\circ} 1$ et la molécule fut donc reprise quelques années plus tard dans des modèles expérimentaux différents de ceux de l'épilepsie. Il fut alors démontré que, comme on l'attendait d'un antiglutamate, le riluzole diminuait la mort neuronale consécutive à l'ischémie [4].
Le site d'action du riluzole fait de ce produit une molécule antiglutamate très particulière. Il ne se lie, en effet, à aucun des récepteurs connus des acides aminés excitateurs, et semblerait intervenir essentiellement au niveau présynaptique en bloquant la libération du glutamate. Des travaux récents indiquent qu'en fait, c'est sur le canal sodique, et, plus spécifiquement, sur l'état inactivé de ce canal, qu'agit le riluzole [5]. La molécule pourrait ainsi interférer indirectement avec la neurotransmission glutamatergique en diminuant l'excitabilité des terminaisons présynaptiques et donc leur efficacité à libérer du glutamate. Cette action indirecte permet, par ailleurs, de mieux comprendre l'absence apparente de toxicité du riluzole puisque, au contraire des autres molécules antiglutamates, il n'interrompt pas les circuits de neurotransmission indispensables au fonctionnement du système nerveux. Les résultats du premier essai clinique dans le traitement de la SLA [1] semblent justifier l'intérêt porté à cette molécule. Dans cette étude chez 155 patients, réalisée en double aveugle, l'effet d'un traitement des patients par riluzole pendant douze mois a été évalué surtout sur la survie et les résultats se sont révélés statistiquement significatifs en faveur du traitement $(74 \%$ contre $58 \%$ de survie). Les données sont même meilleures pour les formes bulbaires, plus rares, puisque la survie est de $73 \%$ contre $35 \%$ à un an. L'effet du traitement est également sensible 
sur la vitesse de détérioration de la fonction musculaire, quoique cet aspect de la maladie n'ait sans doute pas reçu dans cette étude l'attention qu'il mérite.

Le riluzole deviendra-t-il le modèle d'une nouvelle famille de molécules capables de s'opposer efficacement à la mort neuronale dans diverses maladies neurodégénératives? Une étude beaucoup plus large (près de 1000 patients) est actuellement en cours en ce qui concerne la SLA et ses résultats seront déterminants pour l'avenir de la molécule. S'ils sont positifs, il sera permis de rêver au traitement d'autres maladies au pronostic aussi effroyable, notamment la chorée de Huntington pour laquelle la participation d'un phénomène excitotoxique est aujourd'hui considérée comme très probable. La chorée, comme les autres maladies neurodégénératives d'origine génétique, aurait, par ailleurs, l'avantage par rapport à la SLA de permettre un traitement préventif car, si le riluzole - ou d'autres antiglutamates - peut empêcher les neurones de mourir, il est incapable de ressusciter ceux que les malades ont déjà perdus.

M.P.

1. Bensimon G, Lacomblez L, Meininger V, et al. A controlled trial of riluzole in amyotrophic lateral sclerosis. N Eng J Med 1994; 330 : 585-91

2. Mizoule J, Meldrum B, Mazadier M, Croucher M, Ollat C, Uzan A, Legrand IJ, Gueremy C, LeFur G. 2-amino-6-(trifluoromethoxy)benzothiazole, a possible antagonist of excitatory amino acid neurotransmission-I. Anticonvulsant properties. Neuropharmacol $1985 ; 24$ : 767-73.

3. Benavidès J, Camelin JC, Mitrani N, Flamand F, Uzan A, Legrand II, Gueremy C, LeFur G. 2-amino-6-(trifluoromethoxy) benzothiazole, a possible antagonist of excitatory amino acid neurotransmission-II. Biochemical properties. Neuropharmacol 1985 ; 24 : 1085-92. 4. Malgouris C, Bardot F, Daniel M, Pellis F, Rataud J, Uzan A, Blanchard JC, Laduron PM. Riluzole, a novel antiglutamate, prevents memory loss and hippocampal neuronal damage in ischemic gerbils. J Neurosci 1989 ; 9: 3720-7.

5. Benoit E, Escande D. Riluzole specifically blocks inactivated $\mathrm{Na}$ channels in myelinated nerve fibre. Eur J Physiol 1991 ; 419: 603-9.

$\mathrm{m} / \mathrm{s} n^{\circ} 6-7$ vol. 10, juin-juillet 94
Succès de la lutte biologique contre les marées noires. Il y a plus de vingt ans, naissait en laboratoire l'idée que la biodégradation du pétrole pouvait être accélérée par l'addition d'azote et de phosphore. En 1978, l'Amoco Cadiz déversait 220000 tonnes de pétrole sur les côtes bretonnes. A la suite de cette catastrophe, la biodégradation fut reconnue comme mécanisme naturel essentiel de l'élimination des hydrocarbures non volatils de notre environnement. Les micro-organismes qui dégradent les hydrocarbures se retrouvent dans presque tous les écosystèmes et plus de 30 genres de bactéries et de champignons, capables de dégrader les hydrocarbures, ont été identifiés. En 1989, $2000 \mathrm{~km}$ de côtes rocheuses de l'Alaska étaient polluées par les 41000 tonnes de pétrole brut que transportait l'Exxon Valdez Pour accélérer la dégradation naturelle du pétrole, des engrais à base d'azote et de phosphore ont été employés pour stimuler la croissance des micro-organismes locaux. Environ 50 tonnes d'azote et 5 tonnes de phosphore ont ainsi été réparties sur les côtes pendant les mois d'été de 1989 à 1992. En 1989, quelques semaines seulement après le début du traitement, l'agence américaine de protection de l'environnement publiait des images montrant l'efficacité des engrais. Les tests en laboratoire ont confirmé que les engrais ne favorisaient pas l'élimination physique du pétrole et que sa disparition était bien d'ordre biologique. Malheureusement, cela n'a pas pu être confirmé par les techniques de gravimétrie sur le terrain, en partie à cause de l'hétérogénéité des sédiments et des dépôts de pétrole. Une parade a été développée, qui consiste à mesurer, en fonction du temps, certains constituants biodégradables du pétrole et de leurs dérivés, et à les rapporter à un constituant très stable présent en quantité connue au moment de la catastrophe. Grâce à cette méthode, nous savons maintenant que la biodégradation du pétrole de l'Exxon Valdez a été beaucoup plus rapide dans les zones traitées avec les engrais. Les traitements pourront être améliorés en laboratoire en tenant compte de leur toxicité, de la nature des sédiments et de la composition du pétrole. Jusqu'à présent, l'ensemencement de micro-organismes n'a jamais été tenté mais cela pourrait être envisagé dans les zones polluées où ils sont en quantité insuffisante.

[Bragg JR, et al. Nature 1994; 368 : 413-8.]

\section{Les épidermolyses bulleuses} jonctionnelles. Dans un article de médecine/sciences d'avril 1993, Meneguzzi et al. [1] faisaient le point des connaissances sur les épidermolyses bulleuses. Les premières mutations avaient été décrites dans la forme dite simplex et concernaient les kératines 5 (du type II, dont le gène est sur le 12), et 14 (du type I, dont le gène est sur le 17). On incrimina ensuite, dans la forme appelée dystrophique, le collagène VII. Mais, en outre, les auteurs niçois mettaient en cause, dans les formes jonctionnelles, une protéine qu'ils appelaient nicéine, dont le gène est sur le chromosome l en q25-31. Des travaux récents émanant de cette équipe niçoise, associée à des auteurs américains et finlandais [2], et d'auteurs américains avec les mêmes finlandais [3] sont venus confirmer et préciser les données précédentes. Dans la forme d'épidermolyse bulleuse dite jonctionnelle, le clivage du tissu se fait au niveau de la zone dermoépidermique. Ces affections sont autosomiques récessives. Il en existe deux formes cliniques, appelées Herlitz (précoce, généralisée 
et sévère) et non Herlitz. (plus tardive et d'évolution lente). La protéine, suggérée comme candidate dans ces maladies, a été appelée nicéine, épiligrine ou kalinine. Récemment la nomenclature a été unifiée : cette protéine, un hétérotrimère dont les sous-unités $\alpha, \beta$ et $\gamma$ ressemblent à celles de la laminine, a été appelée laminine 5 [4] et ses polypeptides constitutifs sont $\alpha 3, \beta 3, \gamma 2$. On a pu localiser les gènes pour $\alpha 3$ en 18q, pour $\beta 3$ en lq32 et pour $\gamma 2$ en lq25-31. La séquence codante de la sous-unité ү2 (IAMC2) a été analysée et compte 1193 acides aminés [5]. Elle a été étudiée chez des malades atteints d'épidermolyse bulleuse jonctionnelle, dans des familles chez lesquelles l'analyse de liaison désignait comme responsable un locus en lq25-31. Plusieurs mutations ont été caractérisées [2, 3]. (1) Une mutation ponctuelle $\mathrm{G} \rightarrow$ $\mathrm{A}$, avec comme conséquence la délétion d'un exon supprimant $219 \mathrm{pb}$ dans le messager; cette mutation était homozygote. (2) Une délétion de $20 \mathrm{pb}$, suivie de l'insertion d'une seule pb dans l'exon 16 ; il en résulte un décalage de phase et une interruption prématurée ; cette mutation, hétérozygote, laisse à découvrir l'anomalie de l'autre allèle, puisqu'il s'agit d'une maladie récessive. (3) Une mutation homozygote aboutissant à un codon stop K95X. En revanche, dans plusieurs autres familles dont la liaison avec le même locus est significative, aucune mutation n'a encore été détectée. Par ailleurs, dans d'autres familles, ce locus a été exclu, et il faudra analyser en priorité, dès que cela sera possible, les deux autres chaînes de la LAM5. [1. Meneguzzi G, et al. médecine/sciences 1993; 9 : 387-95.]

[2. Pulkinen L, et al. Nature Genet $1994 ; 6: 293-8$.

[3. Aberdam D, et al. Nature Genet $1994 ; 6$ : 299-304.]

[4. Kallunki P, et al. J Cell Biol 1992 ; 119 : 679-93.]

[5. Vailly J, et al. Eur J Biochem 1994; 219 : 209-18.] \\ ] BRÈVES OU}

Nef provoque l'endocytose du CD4. La réplication à titre élevé du virus SIV et l'apparition de l'immunodéficience acquise chez le singe sont liées à la présence de Nef, une protéine précoce spécifique des lentivirus de primates. Nef du VIHl (virus de l'immunodéficience humaine) est une protéine cytoplasmique qui peut se fixer aux membranes par l'intermédiaire d'un acide myristique N-terminal [1]. Le rôle de Nef n'est pas clairement élucidé mais plusieurs travaux ont montré que cette protéine contrôlait négativement l'expression de surface du C.D4, le principal récepteur du virus. CD4 est aussi une molécule primordiale dans l'ontogénie des lymphocytes $\mathrm{T}$ et dans l'activation des cellules $\mathrm{T}$ auxiliaires. p56 $6^{\mathrm{kck}}$, une tyrosine kinase spécifique des cellules lymphoïdes [2], s'associe au domaine intracytoplasmique de CD4 et bloque son internalisation. L'endocytose de C.D4 est observée, cependant, lorsque au cours de la reconnaissance antigénique (.I)4 interagit avec le CMH-II (complexe majeur d'histocompatibilité de classe II) et se sépare de p56 lck. Probablement parce qu'il est à la fois le récepteur viral et une molécule essentielle du système immunitaire, CD4 est une cible privilégiée du VIH. Dans les cellules infectées par VIH, la protéine d'enveloppe gpl60 retient CD4 dans le réticulum endoplasmique où il est dégradé par un mécanisme dépendant d'une autre protéine virale, Vpu. Env et Vpu sont des produits de gènes tardifs synthétisés seulement lorsque l'accumulation de Rev atteint un seuil critique. Ce n'est pas le cas de Nef dont la synthèse est indépendante de Rev et qui peut rapidement exercer son action sur le CD4. Une équipe de chercheurs américains (La Jolla, CA) vient de montrer que Nef induit l'endocytose de CD4 qui est suivie par sa dégradation dans les lysosomes [3]. La myristoylation de Nef et son accrochage à la membrane cellu- laire ne sont pas étrangers à ce phénomène. Dans le domaine intracytoplasmique de C.D4, les 20 acides aminés les plus proches de la membrane contiennent un signal d'endocytose activé par Nef. Deux résidus leucine adjacents sont absolument nécessaires au fonctionnement du signal et se retrouvent dans d'autres protéines, également internalisées et dégradées dans les lysosomes (sous-unités $\gamma$ et $\delta$ du (CD3 par exemple). La région d'interaction de C.D4 avec p56 $6^{\mathrm{kch}}$ n'est pas impliquée dans ce phénomène. Il n'est pas impossible que Nef se lie de façon transitoire au CD4, mais cela reste à démontrer. [1. Boutin J. médecine/sciences 1993 ; 9: 684-92.]

[2. Fagard R, Daniélan S. médecine/sciences $1989 ; 5$ : 564-0.]

[3. Aiken C, et al. Cell 1994; 76 : 853-64.]

La vie des précurseurs
lymphoïdes B, mais non celle des
précurseurs T, est prolongée par
Bcl-2. Chez les souris scid (severe
combined immunodeficiency), les gènes
des immunoglobulines (Ig) et des
récepteurs des antigènes (TcR) ne
sont pas réarrangés normalement.
La lymphopoïèse est défectueuse
car les Ig et les récepteurs des anti-
gènes ne peuvent pas être expri-
més et transmettre les signaux de
survie aux cellules immatures. On
sait que la protéine Bcl-2 augmente
les capacités de survie des
lymphocytes T et B à plusieurs éta-
pes de leur différenciation (m/s
$n^{\circ} 1$, vol. 7 , p. 84 ; $n^{\circ} 4$, vol. 8 ,
$p .392 ; n^{\circ} 10$, vol. 8 , p. $1113 ; n^{\circ} 3$,
vol. 9, p. 328), mais son rôle dans
le développement des cellules pro-
T et pro-B n'avait pas encore été
étudié. C'est maintenant chose
faite grâce à la création de souris
scid, transgéniques pour le gène
bcl-2 s'exprimant dans les cellules
lymphoïdes. Les souris scid ont un
nombre normal de cellules pro-T
et pro-B mais pratiquement pas de
cellules lymphoïdes mûres. Chez


les souris $b c l-2 /$ scid, le nombre de cellules $\mathrm{B} 220+$ (marqueur de différenciation de la lignée B) est élevé et la plupart de ces cellules expriment des marqueurs qui, chez la souris normale, sont caractéristiques des lymphocytes B ayant des Ig de surface. Dans cette population cellulaire, les réarrangements des gènes d'Ig sont plus avancés, sans pour autant être productifs. Les cellules lymphoïdes B de souris scid, malgré l'absence d'Ig de surface, ont donc un phénotype et un génotype plus différencié lorsque $b c l$-2 est présent. Le transgène bcl-2 n'a pas d'effet notable sur le développement des cellules $\mathrm{T}$ chez les souris scid. En revanche, des thymocytes exprimant un récepteur d'antigène (double transgénique $b c l-2 / T c R /$ scid) deviennent sensibles à l'effet de $b c l-2$. Les thymocytes exprimant le récepteur de l'antigène ne sont pas immunologiquement sélectionnés car ils ne reconnaissent pas leur cible dans le contexte génétique de la souris srid. La seule expression d'un récepteur de l'antigène, même en l'absence d'interaction avec le complexe majeur d'histocompatibilité, est donc suffisante pour sensibiliser les thymocytes à l'action de bcl-2. Si l'on suppose que le mode d'action de $b c l-2$ est identique dans les cellules $\mathrm{B}$ et $\mathrm{T}$, ces résultats suggèrent que $b c l-2$ n'est effectif qu'en présence de facteurs qui s'expriment plus ou moins précocement au cours de l'ontogénie des B et des T.

[Strasser A, et al. Nature 1994; 368 : 457-60.]

Des aires corticales sont différenciées avant la mise en place des connexions afférentes. Le néocortex cérébral présente une architecture cellulaire assez homogène, en six couches caractérisées chacune par des populations neuronales identifiables sur une base morphologique. Dès la fin de la phase de développement, toutefois, les aires corticales se différencient les unes des autres par diverses caractéristiques telles que la densité cellulaire, une organisation éventuelle en colonnes, etc. Cette spécification régionale était jusqu'à récemment considérée, pour l'essentiel, comme une conséquence de l'interaction entre des cellules corticales peu spécifiées et des voies de projection, provenant du thalamus, qui imposaient la spécification. Dans une récente nouvelle $\left(\mathrm{m} / \mathrm{s} n^{\circ} \mathrm{l}\right.$, vol. 10, p. 125), nous relations les travaux d'Henry Kennedy et de son équipe (INSERM Lyon, France) montrant que la différence de densité cellulaire entre deux aires voisines du cortex visuel dépendait de mécanismes agissant dans la zone germinative ellemême, ce qui indiquait une spécification préalable à la mise en place de comnexions [1]. Marion Wassef et al. (INSERM et Institut Pasteur, Paris, France) apportent aujourd'hui un autre élément en faveur de cette hypothèse [2]. L'une des lignées de souris transgéniques exprimant le gène LacZ d'E. coli sous le contrôle du promoteur $\mathrm{H}-2 \mathrm{~K}^{\mathrm{b}}$ présente un marquage spécifique des cellules qui forment l'aire somatosensorielle dans le néocortex. Les auteurs ont utilisé cette caractéristique comme un index de spécification pour étudier des transplantations de régions cérébrales foetales chez des hôtes nouveau-nés, une technique qui permet l'intégration des tissus dans les circuits nerveux même en situation ectopique. Ils ont ainsi pu démontrer que cette expression existait quelle que soit la localisation du transplant, c'est-à-dire même lorsque l'aire corticale somatosensorielle génétiquement déterminée était détournée de son activité fonctionnelle et intégrée, par exemple, dans les circuits visuels. A l'inverse, des tissus fotaux comprenant l'aire corticale visuelle ne se mettaient pas à exprimer le transgène lorsqu'ils étaient intégrés en lieu et place de l'aire somatosensorielle. Il existe donc, dans les neurones qui participent à l'aire corticale somatosensorielle, des gènes dont l'expression est spécifique et ne dépend pas de la mise en place des connexions thalamocorticales ou de la mise en route de l'activité nerveuse. De nombreux arguments plaident en faveur d'un rôle de ces afférences et de cette activité dans le modelage des structures nerveuses-cibles au cours du développement. Les résultats d'Henry Kennedy et ceux de Marion Wassef indiquent toutefois que ce modelage intervient sur un patron déjà bien dégrossi.

[1. Dehay C, et al. Nature 1993; 366 : 464-6.]

[2. Cohen-Tannoudji $\mathrm{M}$, et al. Nature 1994; 368 : 460-3.]

Thromboses veineuses, protéine $\mathbf{C}$ et facteur $\mathbf{V}$. La protéine C. activée (APC) est une protéase à sérine dont les puissantes propriétés anticoagulantes sont dues au fait qu'elle inactive les facteurs $\mathrm{V}$ activé et VIII activé (Va et VIIIa). Nous rapportions récemment la fréquence d'une faible réponse anticoagulante à l'APC (20) \% à $50 \%$ ) trouvée chez les malades qui thrombosent $\left(\mathrm{m} / \mathrm{s} n^{\circ} 2\right.$, vol. 10, p. 231). Un défaut qui soustend cette anomalie biologique vient d'être mis à jour. Il s'agit d'une mutation ponctuelle dans le gène du facteur V (169l G $\rightarrow$ A) qui entraîne la production d'un facteur Va (506Arg $\rightarrow$ Gln) dont l'activité n'est plus inhibée par l'APC [1]. La fréquence de cette mutation, retrouvée chez $2 \%$ à $4 \%$ de la population hollandaise et $7 \%$ de la population suédoise, fait s'interroger sur l'avantage sélectif qu'elle pourrait apporter [2]. Il est vraisemblable que d'autres mutations du facteur V peuvent donner le même tableau d'un facteur $V$, activable en Va, mais dont l'activité coagulante ne peut être inhibée par l'APC.

[1. Bertina RM, et al. Nature 1994 ; 369 : 64-7.]

[2. Majerus P. Nature 1994; 369 : 14-5.] 